NASA/TM-1999-209295

D.

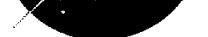

\title{
Multiple Point Dynamic Gas Density Measurements Using Molecular Rayleigh Scattering
}

Richard Seasholtz

Glenn Research Center, Cleveland, Ohio

Jayanta Panda

Modern Technologies Corporation, Middleburg Hts., Ohio

Prepared for the

18th International Congress on Instrumentation in Aerospace Simulation Facilities Conference cosponsored by the Office National d'Études et de Recherches Aérospatiales and the Institute of Electrical and Electronics Engineers

Toulouse, France, June 14-17, 1999

National Aeronautics and Space Administration

Glenn Research Center 


\section{Acknowledgments}

We would like to acknowledge the diligent efforts of Mr. W. Trevor John, who was responsible for setting up the optical system used for this work

Available from

NASA Center for Aerospace Information

7121 Standard Drive

Hanover, MD 21076

Price Code: A03
National Technical Information Service 5285 Port Royal Road Springfield, VA 22100 Price Code: A03 


\title{
MULTIPLE POINT DYNAMIC GAS DENSITY MEASUREMENTS USING MOLECULAR RAYLEIGH SCATTERING
}

\author{
Richard Seasholty \\ National Aeronautics and Space Administration \\ Glenn Research Center \\ Cleveland, $\mathrm{OH} 44135$ \\ Jayanta Panda \\ Modern Technologies Corporation \\ Middleburg Hts., $\mathrm{OH} 44130$
}

\begin{abstract}
A nonintrusive technique for measuring dynamic gas density properties is described. Molecular Rayleigh scattering is used to measure the time-history of gas density simultaneously at eight spatial locations at a $50 \mathrm{kHz}$ sampling rate. The data are analyzed using the Welch method of modified periodograms to reduce measurement uncertainty. Cross-correlations, power spectral density functions, cross-spectral density functions, and coherence functions may be obtained from the data. The technique is demonstrated using low speed co-flowing jets with a heated inner jet.
\end{abstract}

\section{INTRODUCTION}

Quantitative techniques for measurement of fluid flow properties in aerospace test facilities are needed to obtain performance data. to assess the performance of new computer codes, and to establish facility flow conditions. In many test programs, conventional probe measurements are not adequate because they perturb the flow under study to an intolerable degree. This is a particularly severe problem in supersonic flows, where physical probes generate shocks and cause large changes in the flow patterns. In turbulent flows, the presence of a probe will alter the turbulence characteristics.

In this paper, we describe a flow diagnostic technique based on the molecular Rayleigh scattering of laser light. The objective of the work is to develop an unseeded, nonintrusive dynamic measurement technique for the study of turbulent flows in NASA test facilities. This technique will provide aerothermodynamic data that are not presently available. These data will be important in studies such as the growth and decay of turbulent fluctuations. The effort is part of the non-intrusive instrumentation development program supporting supersonic and hypersonic propulsion research at the NASA Glenn Research Center. In particular, this work is directed to the measurement of fluctuations in flow velocity, density. and temperature.
One of the main objectives in jet noise research is to identify noise sources in the jet and to determine their contribution to noise generation. In particular, researchers have focused on the correlation of fluctuations in flow parameters with far field noise [1]. In this work. we describe a nonintrusive technique for measuring gas density fluctuations. Density time-history is measured and used to obtain the power spectrum of the density fluctuations. Simultaneous multiple point measurements are used to obtain density cross-correlations and crossspectra. The technique is based on the simplest application of Rayleigh scattering. where only the total intensity of the Rayleigh scattered signal is measured. The gas density is proportional to the total scattered light. Because the Rayleigh scattering cross sections is a function of the molecular species that make up the flow, knowledge of the gas composition is generally required; however, this is not a concern in this work, which is directed toward air flows where the composition is well defined. Although this basic technique has been used by various researchers. including us, in this paper we extend the technique to the simultaneous measurement of density at a number of locations with a high frequency response $(10 \mathrm{kHz})$. The resulting measurements allow the computation of the density power spectrum at each of the measurement locations. In addition, the cross correlations and cross power spectra can also be obtained. An important practical feature of Rayleigh scattering is that because it is a molecular scattering process, no seeding of the flow is needed. In fact, the elimination of particles in the flow is a requirement for accurate density measurements.

We have previously measured time-average and phaseaverage density in a screeching jet [2] and used spectrally resolved Rayleigh scattering to measure time-averaged density, velocity, and temperature in a underexpanded supersonic, shock-containing free jet [3]. We also measured density fluctuation spectra in fully expanded supersonic jets with Mach numbers 1.4 and 1.8 [4].

In other work, LDV has heen used to measure velocity power spectrum [5]. Rayleigh scattering has been used 
(with LDV) to measure mean density in supersonic, hightemperature flow [6]. Two pulsed lasers were used to measure spatial and temporal correlations in an underexpanded supersonic free jet [7].

We have previously examined a scheme for dynamic measurement of velocity and temperature using spectrally resolved Rayleigh scattering [8] at a single point. Our eventual goal is to measure space-time correlations of quantities of interest in basic studies of jet noise. In particular, we plan to measure two-point correlations of $\rho V^{2}$ (the product of density and velocity squared). These data will be used to relate properties of turbulent flow to emitted acoustic noise.

In this paper, we discuss the application of molecular Rayleigh scattering for simultaneous, multiple-point dynamic gas density measurements. The multiple channel time history records are used to calculate probability density functions (pdfs), power spectral density functions, crosscorrelation functions, cross-spectral density functions, and coherence functions. The effect of photon shot noise, stray laser light scattering, and particle scattering are discussed. Experimental data are presented for measurements taken in a hot, low-speed free air jet to illustrate the technique.

\section{THEORY}

\section{Rayleigh Scattering}

For a gas with density $\rho$ illuminated by a laser beam with wavelength $\lambda_{1}$ and power $P_{0}$, the expected count rate of detected photoelectrons resulting from light scattered into solid angle $\Omega$ is

$$
\lambda_{R}=\frac{\varepsilon P_{n} \rho L_{1} \lambda_{n} \Omega}{m h c}\left(\frac{\mathrm{d} \sigma}{\mathrm{d} \Omega}\right) \sin ^{2} \chi
$$

where $L_{1}$ is the length along the beam of the scattering volume. $\mathrm{d} \sigma / \mathrm{d} \Omega$ is the Rayleigh differential scattering cross section [9], and $\chi$ is the angle between the electric field vector of the (linearly polarized) incident light and the direction of the scattered light. Generally experiments are arranged so the incident electric vector is perpendicular to the scattering plane (i.e., "s" type) and $\chi=90^{\circ}$; for this situation the scattering is independent of the scattering angle. However, if the electric vector is in the scattering plane (i.e., " $p$ " type), the scattering is dependent on the scattering angle and approaches zero at a $90^{\circ}$ scattering angle. In this equation, $\varepsilon$ is the overall collection efficiency (including the detector quantum efficiency), $h$ is Planck's constant, $c$ is the velocity of light, and $m$ is the molecular mass.
Since Rayleigh scattering is a relatively weak process, the uncertainty in the measurements often is set by the photon statistical noise (shot noise), which determines the lower bound on measurement uncertainty. For example, the variance in the number of photoelectron counts for a Poisson process is equal to the mean number of counts. Thus the lower bound for the relative uncertainty in the measurement of gas density $\rho$, is equal to the square root of the variance divided by the mean counts.

$$
\frac{\sigma(\rho)}{\rho}=\left(\frac{1}{\lambda_{R} \Delta t}\right)^{1 / 2}
$$

where $\Delta t$ is the measurement time interval.

As an example of the expected scattering intensity, about 40 million photoelectrons/sec would be detected by an $f / 4$ optical system with a collection efficiency of $5 \%$ from a $0.8 \mathrm{~mm}$ long probe volume of nitrogen at a pressure of $1 \mathrm{~atm}$ and a temperature of $293 \mathrm{~K}$ irradiated by a $5 \mathrm{~W}$. $532 \mathrm{~nm}$ laser beam. This high photoelectron rate means that independent density measurements could be obtained at a high rate. If we obtain 10,000 independent samples/sec, the mean number of detected photoelectrons would be about 4000 , and the corresponding uncertainty in each density measurement would be $2 \%$. Note that this lower bound represents the situation with no additional noise sources. In practice, one often encounters addition noise from ambient background light, stray laser light. and scattering from particles in the flows.

\section{Statistical description of dynamic Rayleigh scattering}

The molecular Rayleigh scattering from a turbulent fluid can be considered as a doubly stochastic process becausc it includes both the random nature of the fluid density, as well as the Poisson statistics of the light detection process [10]. Because of the finite bandwidth of any real system. the detection process can be modeled as a sequence of impulses filtered by a linear filter. Consider a total detected photon count rate $\lambda(t)=\lambda_{R}(t)+\lambda_{W}(t)$, where $\lambda_{R}(t)$ is duc 10 Rayleigh scattering, and $\lambda_{W}(t)$ is due to other sources. If the light detection system frequency response is $H(f)$, then the measured one-sided power spectral density function of the output can be written

$$
\hat{G}_{m}(f)=\left[2 \bar{\lambda}_{w^{\prime}}+2 \bar{\lambda}_{R}+\hat{G}_{\lambda_{R}}(f)\right]|H(f)|^{2}
$$

where $\hat{G}_{\lambda_{k}}(f)$ is the spectral density function of the fluid density fluctuations. (Here we assume that the mean has been subtracted from the signal to avoid a large component at $f=0$.) As shown in figure 1 , the measured power spectral 
density is the desired spectrum due to density fluctuations added to a constant level due to shot-noise.

Another potential source of noise is a result of Mie scattering from particles in the flow. The transit time of a particle with velocity $V$ crossing a probe volume with width $w$ is $\tau=w / N$. For a top-hat detector time response with amplitude $A$, the two-sided spectral density function is

$$
S_{p}(f)=\left(A \tau \frac{\sin \pi f \tau}{\pi f \tau}\right)^{2}
$$

which has a $3 \mathrm{~dB}$ point (half power) $f_{3, l}=0.44 / \tau$.

In addition to the direct scattering from particles passing through the probe volume, a smaller signal can also be generated by Mie scattering from particles passing through the laser beam outside the direct field of view of the detector. The light scattered from these particles can reach the detection optics by means of multiple scattering from surfaces in the field of view.

For many applications, the desired information is expressed as statistical quantities such as correlation functions and power spectra. For simultaneous, multiple-point measurements one can ohtain additional quantities including cross-correlation functions, cross-spectra, and coherence functions. Consider the time history of two stationary random processes $x(t)$ and $y(t)$, representing density measurements at two locations. The mean, variance, and covariance are [1]]

$$
\begin{aligned}
& \mu_{x}=E[x], \quad \mu_{y}=E[y] \\
& \sigma_{x}^{2}=E\left[\left(x-\mu_{x}\right)^{2}\right], \sigma_{y}^{2}=E\left[\left(y-\mu_{y}\right)^{2}\right] \\
& \sigma_{x y}=E\left[\left(x-\mu_{x}\right)\left(y-\mu_{y}\right)\right]
\end{aligned}
$$

Note that these quantities do not depend on the time history of the fluctuations. but only on their probability distribution functions.

Dynamic quantities, such a correlation functions and spectra, require the actual time histories of the data. We can then define the covariance function

$$
\begin{aligned}
& C_{n}(\tau)=E\left[\left\{x(t)-\mu_{x}\right\}\left\{y(t+\tau)-\mu_{y}\right\}\right] \\
& =\lim _{T \rightarrow \infty} \frac{1}{T} \int_{(1}^{T}\left\{x(t)-\mu_{x}\right\}\left\{y(t+\tau)-\mu_{y}\right\} d t
\end{aligned}
$$

where the correlation function is

$$
R_{\mathrm{v}}(\tau)=\lim _{\tau \rightarrow \infty} \frac{1}{T} \int_{0}^{T} x(t) y(t+\tau) d t
$$

which is related to the covariance function

$$
R_{x y}(\tau)=C_{x y}(\tau)+\mu_{s} \mu_{y}
$$

It is convenient to remove the mean from the data before processing. In this case $R_{x y}(\tau)=C_{n}(\tau)$. For the special case of $x=y, R_{u}(\tau)$ is the autocorrelation function.

The two-sided cross-spectral density function is defined as the Fourier transform of the correlation function

$$
S_{v y}(f)=\int_{-\infty}^{\infty} R_{v y}(\tau) e^{-i 2 \pi \tau \tau} d \tau, \quad-\infty<f<\infty
$$

Note that $S_{k, f}(-f)=S^{*},(f)=S_{n,}(f)$

In presenting experimental results it is common to use the one-sided spectrum $G_{n}(f)=2 S_{n}(f), f>0$

The one-sided cross-spectrum, which is complex, can written in terms of its real and imaginary parts

$$
G_{n v}(f)=2 \int_{-\infty}^{\infty} R_{n}(\tau) e^{-i 2 \pi t \tau} d \tau=C_{n}(f)-i Q_{n}(f)
$$

where $C_{x r}(f)$ is the coincident spectral density function (cospectrum), and $Q_{n}(f)$ is the quadrature spectral density function. These can also write in terms of magnitude and phase

$$
\begin{aligned}
& \left|G_{n}(f)\right|=\sqrt{C_{n}^{2}(f)+Q_{n}^{2}(f)} \\
& \theta_{n}(f)=\tan ^{-1}\left[\frac{Q_{n}(f)}{C_{n}(f)}\right]
\end{aligned}
$$

The magnitude squared normalized by the product of the spectral density function is known as the coherence function

$$
\gamma^{2} w(f)=\frac{\left|G_{u v}(f)\right|^{2}}{G_{u}(f) G_{u}(f)}
$$

which has a range $[0,1]$. The coherence function is a powerful tool that finds use in a variety of applications. Cross-spectrum analysis offers the advantage over crosscorrelation analysis in that information may be obtained even if the propagation is dispersive [11]. 


\section{Welch method of modified periodograms}

We use the Welch method of modified periodograms[12] to calculate the power spectral density (PSD) and cross spectrum density. In this procedure, a long data record sampled at rate $f_{s}$ for time $T$ (total samples $=N=T f_{s}$ ) is subdivided into a number $K$ of smaller records (which may be overlapping), each of length $L$ samples. The modified periodgrams of each subrecord are calculated using a data window, which are then averaged to obtain the estimate of the power spectral density. The frequency resolution of the resulting spectral density is thus $f_{s} / L$. By overlapping the segments by one half of their length, a near maximum reduction in the variance in the PSD estimate is achieved: the variance in the estimated spectral density is reduced by a factor of $11 / 9 \mathrm{~K}$ compared to the variance of a single spectral estimate. The modified periodiogram of the $k^{\text {th }}$ subrecord is given by

$$
I_{k}\left(f_{n}\right)=\frac{L}{U}\left|A_{k}(n)\right|^{2}
$$

where $f_{n}=n / L$ for $\mathrm{n}=0 \ldots \mathrm{L} / 2$, and

$$
\begin{gathered}
A_{k}(n)=\frac{1}{L} \sum_{j=0}^{l-1} X_{k}(j) W(j) e^{-2 k i j n / l} \\
\text { for } k=1.2, \ldots K
\end{gathered}
$$

and

$$
U=\frac{1}{L} \sum_{j=0}^{L-1} W^{2}(j)
$$

with $W(j)$ being the window function. (In our work we used the Bartlett window.)

The spectral estimate is the average of these modified periodiograms

$$
\hat{P}\left(f_{n}\right)=\frac{1}{K} \sum_{k=1}^{K} I_{k}\left(f_{n}\right)
$$

The estimates of the cross spectral density are obtained in a similar manner.

\section{Setup}

\section{EXPERIMENT}

The flow used to demonstrate the multiple-point Rayleigh scattering technique consisted of a $12 \mathrm{~mm}$ diameter electrically heated free jet with a $52 \mathrm{~mm}$ diameter ambient temperature co-flow (fig. 2). The mixing of two flows of different temperatures creates fluctuations in the density. The air supplied to both flows was filtered using a threestage filtration system to remove particles and oil vapor. Stainless steel screens were placed in both flows to reduce flow non-uniformity. The inner heated jet exit extended $15 \mathrm{~mm}$ beyond the co-flow exit plane. A type $\mathrm{K}$ thermocouple and total pressure probe were used to determine the mean flow properties along the jet axis.

The beam from a diode-pumped, solid-state, frequencydoubled, Nd:Vanadate laser with $5 \mathrm{~W}$ of output power at $532 \mathrm{~nm}$ was directed along the jet axis and into the inner nozzle, which served as the beam dump. The diameter of the beam at the measurement region was about $1 \mathrm{~mm}$.

The Rayleigh scattered light was collected with a $50 \mathrm{~mm}$ focal length, $f / 1.2,35-\mathrm{mm}$ camera lens at a $90^{\circ}$ scattering angle and focused onto a multiple anode photomultiplier tube. This tube consists of a 32 channel linear array; with each photocathode having an active area $0.8 \mathrm{~mm}$ wide $x$ $7 \mathrm{~mm}$ high. The height of the active area was limited (to reduce detection of stray light) to $3 \mathrm{~mm}$ by a mask placed in front of the detector. The distance between photocathode active areas is $1 \mathrm{~mm}$. For this work, we used alternate photocathodes to increase the separation between probe volumes. The quantum efficiency given by the manufacturer at $532 \mathrm{~nm}$ is about $8 \%$, and the anode pulse rise time is $0.2 \mathrm{~ns}$.

The positions of the eight probe volumes (set by the magnification of the light collection lens) was measured by scanning a $1 \mathrm{~mm}$ diameter aperture, illuminated by diffuse laser light, through the measurement region. The results of these measurements showed that the separation between probe volumes was $3.82 \mathrm{~mm}$.

\section{Data Acquisition}

Only cight channels of the multiple-anode PMT were used in this work because of the limited number of A/D channels in the data acquisition system. The output of each PMT channel was terminated in a $10 \mathrm{k} \Omega$ load resistor. The maximum allowable PMT mean current/channel of $10 \mu \mathrm{A}$ is thus converted to $0.1 \mathrm{~V}$. A personal computer based data acquisition system was used to digitize the eight analog signals. The combination of the load resistors and the PMT capacitance results in a limited bandwidth. Data were taken on a single channel at a $400 \mathrm{kHz}$ sampling rate to determined the system frequency response. As shown on figure 3 , the $3 \mathrm{~dB}$ cutoff frequency is about $10 \mathrm{kHz}$, with a rolloff of about $20 \mathrm{~dB} /$ decade. Ideally, because of the slow rolloff of this first order RC filter, anti-aliasing filters should be used. However, for this work, we lacked a sufficient number of filters. Thus, with the $50 \mathrm{kHz}$ sampling rate that was used, there will be a small amount of noise aliased from 
higher frequencies into the $0-25 \mathrm{kHz}$ frequency band. The laboratory was darkened during the data acquisition to eliminate the detection of any ambient light. Also, the A/D system does not actually sample the eight channels simultaneously, but instead samples them sequentially. Thus, there is a time separation of $2.5 \mu$ s between samples from adjacent channels. However, this should not result in significant errors for the frequencies of interest in this study $(<10 \mathrm{kHz})$.

The relative response of the eight PMT channels was determined using the spatially uniform light source generated by a $200 \mathrm{~mm}$ diameter integrating sphere. Low level laser light was introduced into the sphere. Figure 4 shows the resulting power spectrum for each of the eight channels. For this test, channel number 1 included a $10 \mathrm{kHz}$ low-pass anti-aliasing filter to evaluate its performance. The other channels did not include a filter. A $50 \mathrm{kHz}$ sampling rate was used with 200,000 samples taken for each channel. The response of the eight channels was reasonably uniform, with the standard deviation from the mean being less than $8 \%$. Note that the response of the channel with the anti-aliasing filter was reduced by a factor of two relative to the other channels.

\section{Results}

\section{Measurements along flow axis}

Data were taken along the axis of the low-speed heated air jet, with the imaged region being located at two positions. In the first region, the ratio of the distance from the jet exit plane to the jet diameter $(X / D)$ ranged from 1.4 to 3.6 . In the second region $X / D$ ranged from 3.1 to 5.3 . At each of these positions, eight channels of Rayleigh scattering data were obtained at a $50 \mathrm{kH} z$ sampling rate. The data acquisition time was 4 seconds, yielding 200,000 samples for each channel.

Data were taken at a number of flow conditions, with the inner jet exit plane temperature at ambient temperature, $200^{\circ}, 300^{\circ}, 400^{\circ}, 500^{\circ}$, and $600^{\circ} \mathrm{C}$. Here, we only show data taken at ambient temperature and at $600^{\circ}$. The power spectral density estimates obtained using the Welch method of modified periodograms described above for the eight channels in the two test regions are shown in figures 5 and 6 . For these power spectra estimates, the subrecord length was 2048 , so the frequency resolution was $24.4 \mathrm{~Hz}$.

Figure 5 shows the spectral estimates for the inner jet at the same temperature as the outer coflow. The velocity at the inner jet exit plane, determined from a total pressure measurement, was about $8 \mathrm{~m} / \mathrm{sec}$. As, expected these show very little density fluctuations. The small increase in the spectral density at low frequencies may be caused by secondary light scattering from particles in the laser beam outside the measurement region. Or, it may have been the result of scattering from a small number of residual particles in the flow.

Figure 6 shows the power spectral estimates of the density for data taken at an inner jet exit temperature of $600^{\circ} \mathrm{C}$ and a exit velocity of $19 \mathrm{~m} / \mathrm{sec}$. The spectra exhibit very large values at low frequencies, about two orders of magnitude above the spectra for unheated flow. The bandwidth of density fluctuations is about $2 \mathrm{kHz}$.

Information on the flow evolution can be obtaining by using the simultaneous multiple channel data. The crosscorrelation between adjacent channels (such as shown figure 7) gives a measure of the convective velocity of turbulent structures in the flow. For channels 1 and 2 $(X / D=3.1,3.4)$, the delay of the cross-correlation peak is $0.5 \mathrm{msec}$. Using the $3.8 \mathrm{~mm}$ channel spatial separation gives a $7.6 \mathrm{~m} / \mathrm{sec}$ convective flow velocity. Figure 8 shows the convective velocity determined from crosscorrelation for seven locations in each of the two measurement regions.

More information can be obtained by calculating the cross spectra. For example, the phase of the cross spectrum between channels 1 and 2 for the $600^{\circ} \mathrm{C}$ flow is shown in figure 9. The linear slope indicates a non-dispersive phenomenon, and the velocity calculated from the $0.180 \% \mathrm{~Hz}$ slope is $7.6 \mathrm{~m} / \mathrm{sec}$, the same as found from the cross-correlation function. The advantage of the crossspectrum analysis is that dispersive phenomena can also be studied.

The other quantity of interest that can be obtained from the cross spectrum is the coherence function (as discussed above). Figure 10 shows the coherence functions calculated for one of the measurement regions between channel $1(X / D=1.4)$ and the other 7 channels (at $X / D=$ $1.7,2.0,2.4,2.7,3.0,3.3$, and 3.6) with $600^{\circ} \mathrm{C}$ flow. Note the appearance of density fluctuations in figure $10 \mathrm{~b}$ with frequencies of about $1 \mathrm{kHz}$ for the coherence function between $X / D=1.4$ and 2.0. This peak occurs at lower frequencies at wider separations, which is probably a result of the slowing of the flow as shown in figure 8. The subrecord length used for these coherence functions was 512 , resulting in a frequency resolution of $97.7 \mathrm{~Hz}$.

\section{CONCLUDING REMARKS}

This preliminary work demonstrates the potential of molecular Rayleigh scattering as a technique to study the dynamic behavior of turbulent flows. For flows with stationary turbulence parameters, the relatively weak Rayleigh scattering can be analyzed by taking long record 
lengths and then averaging using the Welch method of modified periodograms to reduce the variance of spectral estimates. Although the work presented here used low speed co-flowing jets with the inner jet heated to generate density fluctuations. the technique can be extended to high speed compressible flows which have large density fluctuations related to the flow turbulence. Furthermore, we plan to extend the Rayleigh scattering dynamic measurements to include velocity using a previously developed technique based on resolving the Rayleigh spectrum with a Fabry-Perot interferometer. The goal is to measurement correlations of the product of density and velocity at two or more locations. This will be a powerful new tool for studies of the generation of noise by supersonic jets. It will be of particular importance because it is a non-intrusive technique. Use of any physical probe measurements in high speed flows provides questionable data, at best. because the flow under study is altered by the probe.

\section{REFERENCES}

11] Schaffar, M., "Direct measurements of the correlation between axial in-jet velocity fluctuations and far-field noise near the axis of a cold jet", J. Sound and Vibration, Vol. 64, pp. 73-83, 1979.

12) Panda, J. and R.G. Seasholtz, "Density measurement in underexpanded supersonic jets using Rayleigh scattering". AIAA $36^{\text {th }}$ Aerospace Sciences Meeting, Reno, NV, AIAA-98-0281, 1998.

[3] Panda. J. and R.G. Seasholtz, "Velocity and temperature measurement in supersonic free jets using spectrally resolved Rayleigh scattering", AlAA $37^{\text {hi }}$ Aerospace Sciences Meeting, Reno. NV, AIAA-99-0296, 1999.

[4] Panda, J.. and R.G.Seasholtz, "Density fluctuation measurement in supersonic fully expanded jets using Rayleigh scattering", $5^{\text {th }}$ AIAA/CEAS Aerocoustics Conference, Seattle, WA. NV, AIAA-99-1870, 1999.
[5] Nobach, H., E. Muller, and C. Tropea, "Efficient estimation of power spectra density from laser Doppler anemometer data", Exp. Fluids, Vol, 24, pp. 499-509, 1998.

[6] Jiang, L.Y., and J.P. Sislian, "Velocity and density measurements in supersonic high-temperature exhaust plumes", AIAA J., Vol. 36, pp. 1216-1222, 1998.

[7] Nerushev. O.A., S.A. Novopashin, and A.L. Perepelkin, "Rayleigh light scattering as a pulse local probing of non-equilibrium gas flows". ICIASF '97 Record, F.K. Owen, Ed., pp. 336-342, 1997.

[8] Seasholtz, R.G.; and J. Panda, "Rayleigh scattering diagnostic for dynamic measurement of velocity and temperature", AIAA $37^{\text {th }}$ Aerospace Sciences Meeting, Reno, NV, AIAA-99-0641, 1999.

[9] Gardiner, W.C. Jr., Y. Hidaka, and T. Tanzawa, "Refractivity of Combustion Gases", Comb. and Flame, Vol. 40, pp. 213-219, 1981.

[10] Goodman, J.W., Statistical Optics, John Wiley \& Sons, New York, pp. 85-98, 1985.

111] Bendat, J.S., and A.G. Piersol, Engineering Applications of Correlation and Spectral Analysis, John Wiley \& Sons, New York, 1980.

[12] Welch, P.D., "The use of fast Fourier transform for the estimation of power spectra: A method based on time averaging over short, modified periodograms", IEEE Trans. on Audio and Electroacoustics, Vol. AU-15, pp. 70-73. 1967. 


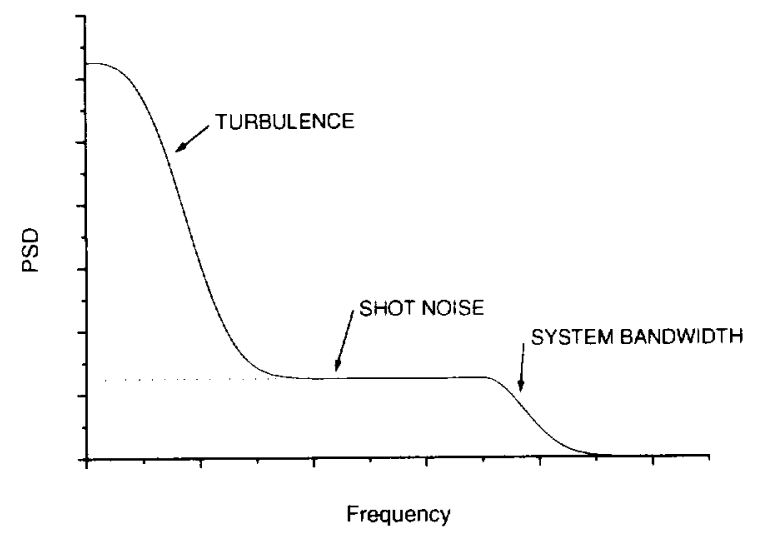

Fig. 1 - Expected spectrum for Rayleigh scattering density measurements in turbulent flow. The dc component of the signal is not shown. The shot noise floor is due to the photon statistical noise and is proportional to the mean signal level.

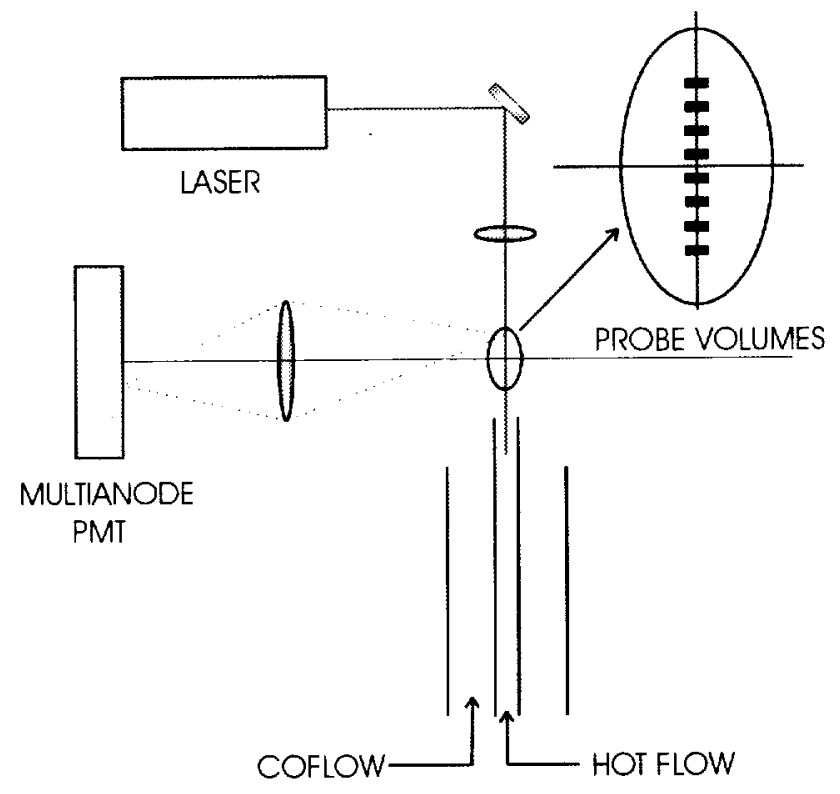

Fig. 2 - Experiment to measure density fluctuations at eight locations along axis of heated free jet. Each probe volume, defined by the image of the active area of the PMT photocathode and the laser beam width, is $1.53 \mathrm{~mm}$ wide hy $1 \mathrm{~mm}$ high. The separation between probe volumes is $3.82 \mathrm{~mm}$.

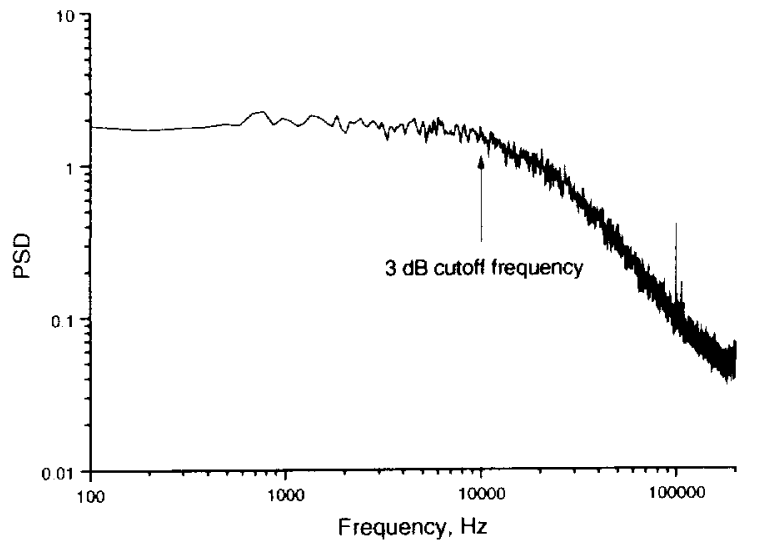

Fig. 3 - Single channel of data sampled at $400 \mathrm{kHz}$. This shows that the $3 \mathrm{~dB}$ cutoff frequency is about $10 \mathrm{kHz}$ when no additional low pass filtering is used.

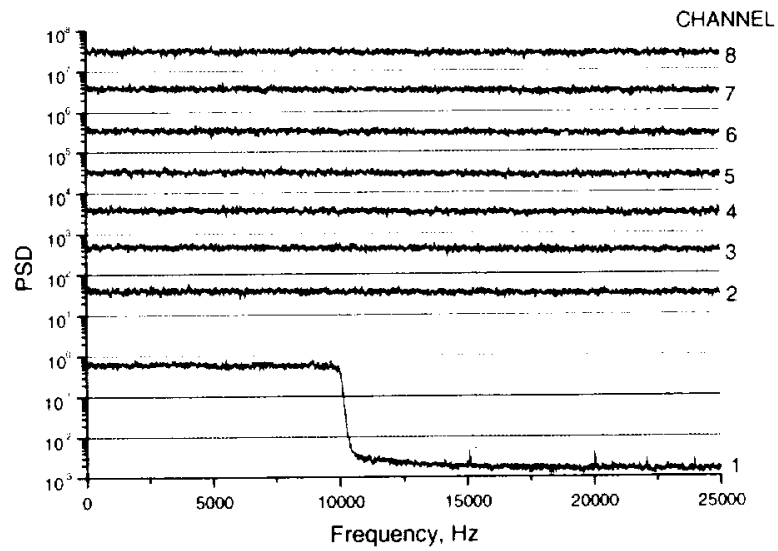

Fig. 4 - Power spectrum of laser light from integrating sphere. Channel 1 includes $10 \mathrm{kHz}$ antialiasing low pass filter: level is reduced by factor of two for this channel relative to the other channels. Channels are offset by powers of 10 . 


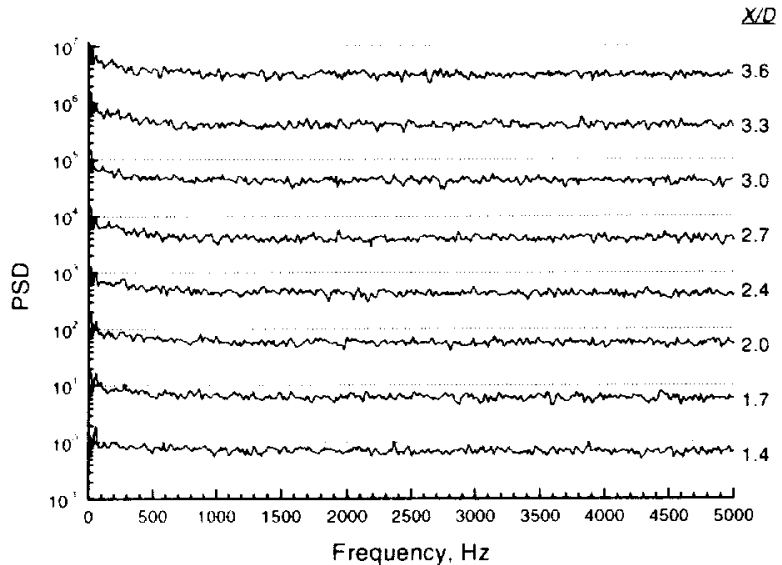

(a)

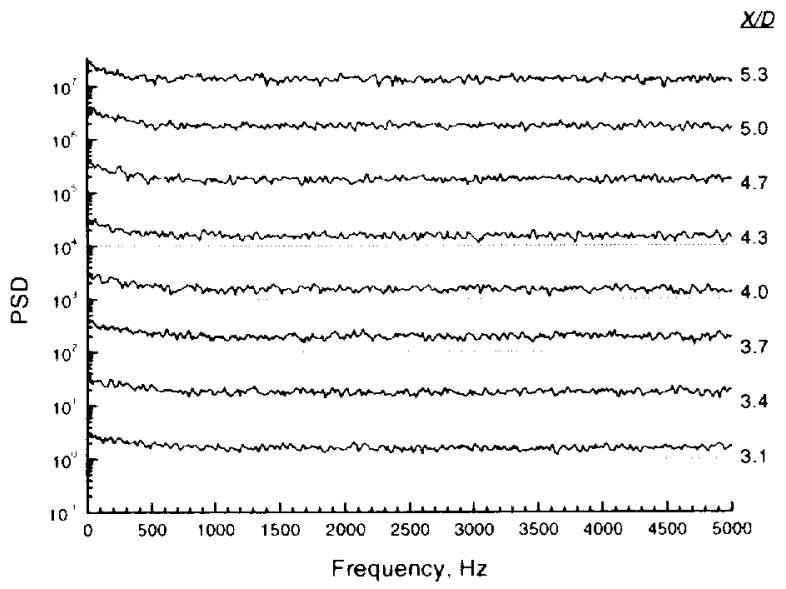

(b)

Fig. 5- Simultaneous power spectral density for cold flow at eight axial locations (a) $X / D$ from 1.4 to 3.6. (b) X/D from 3.1 to 5.3. Spectra are offset by powers of 10. Based on 200,000 samples for each channel with $50 \mathrm{kHz}$ sampling rate.

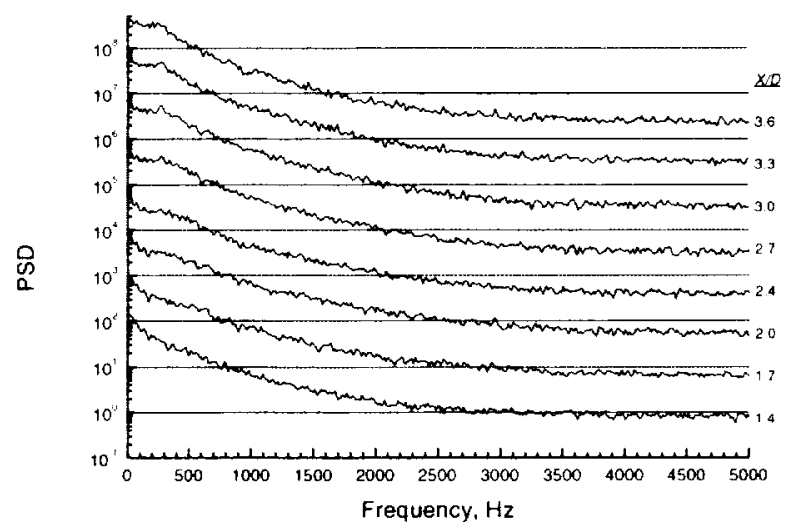

(a)

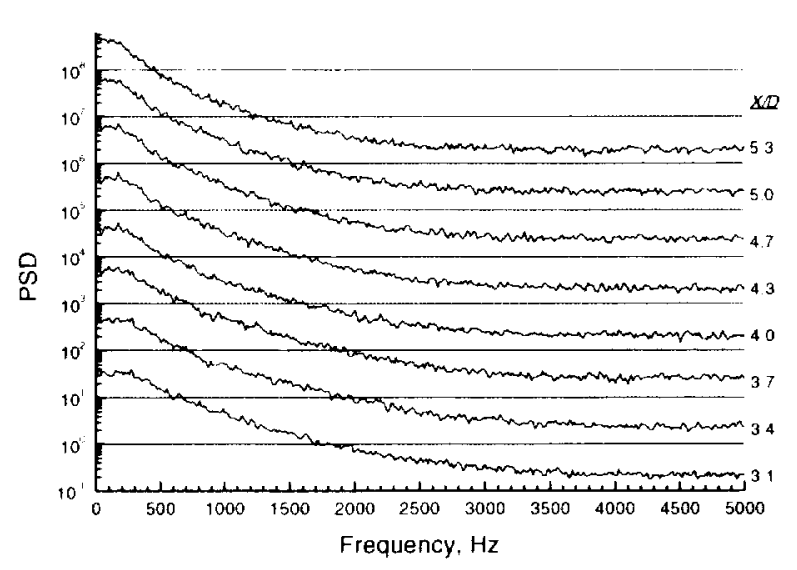

(b)

Fig. 6 - Simultaneous power spectral density at eight axial locations (a) $X / D$ from 1.4 to 3.6, (b) $X / D$ from 3.1 to 5.3. Exit temperature is $600^{\circ} \mathrm{C}$. Spectra are offset by powers of 10 . Based on 200,000 samples for each channel with $50 \mathrm{kH} z$ sampling rate. 


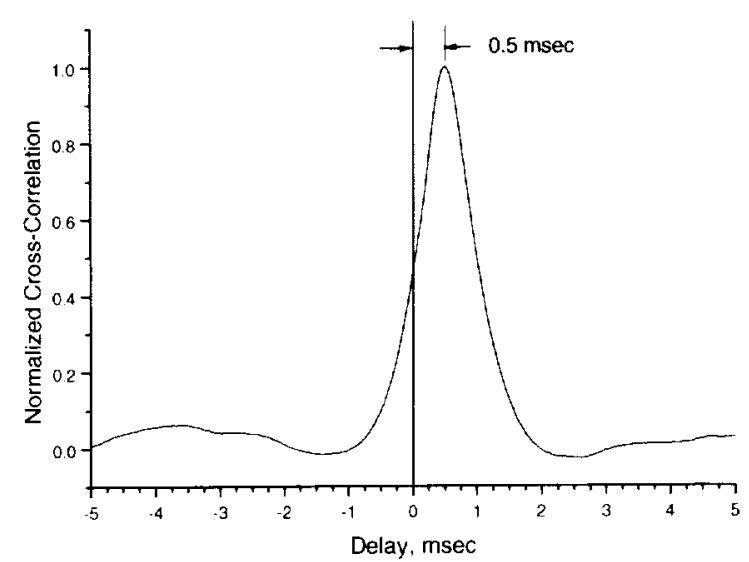

Fig. 7 - Cross-correlation of channels 1 and 2, located at $X / D=3.1$ and 3.4. Corresponding velocity is 3.82 $\mathrm{mm} / 0.5 \mathrm{msec}=7.6 \mathrm{~m} / \mathrm{sec}$.

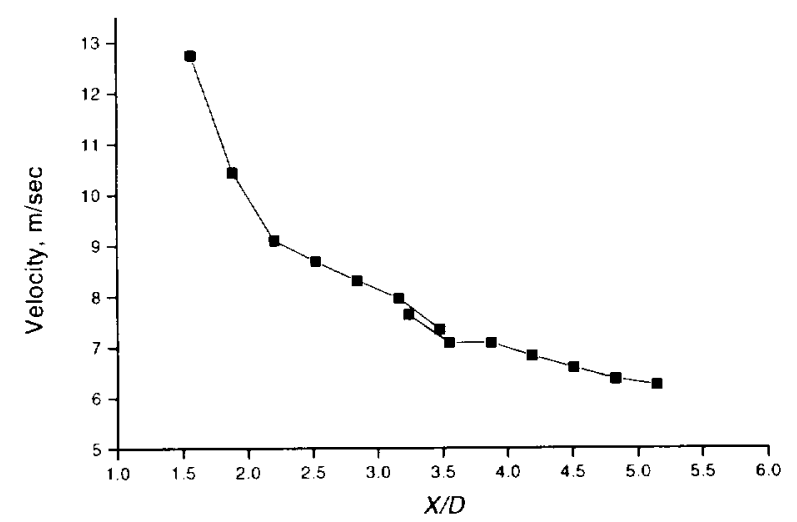

Fig. 8 - Velocity calculated from cross-correlation of adjacent channels. $X / D$ based on mean of two channel positions. Jet exit temperature $=600^{\circ} \mathrm{C}$.

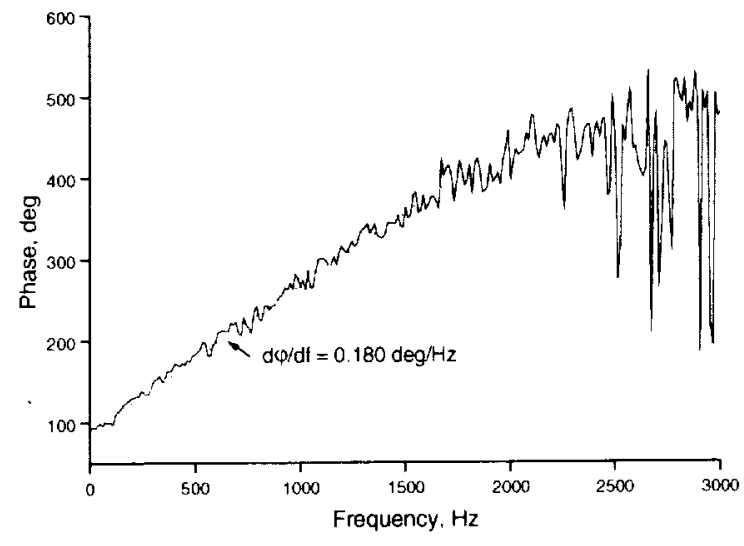

Fig. 9 - Cross spectrum phase for channels 1 and 2, $X / D=$ 3.1 and 3.4 for jet exit temperature of $600^{\circ} \mathrm{C}$. Slope for the frequency ranage of 0 to $2000 \mathrm{~Hz}$ is $0.180^{\circ} / \mathrm{Hz}$.

Corresponding velocity is $7.6 \mathrm{~m} / \mathrm{sec}$ (same as given by cross correlation shown in figure 7 ). 

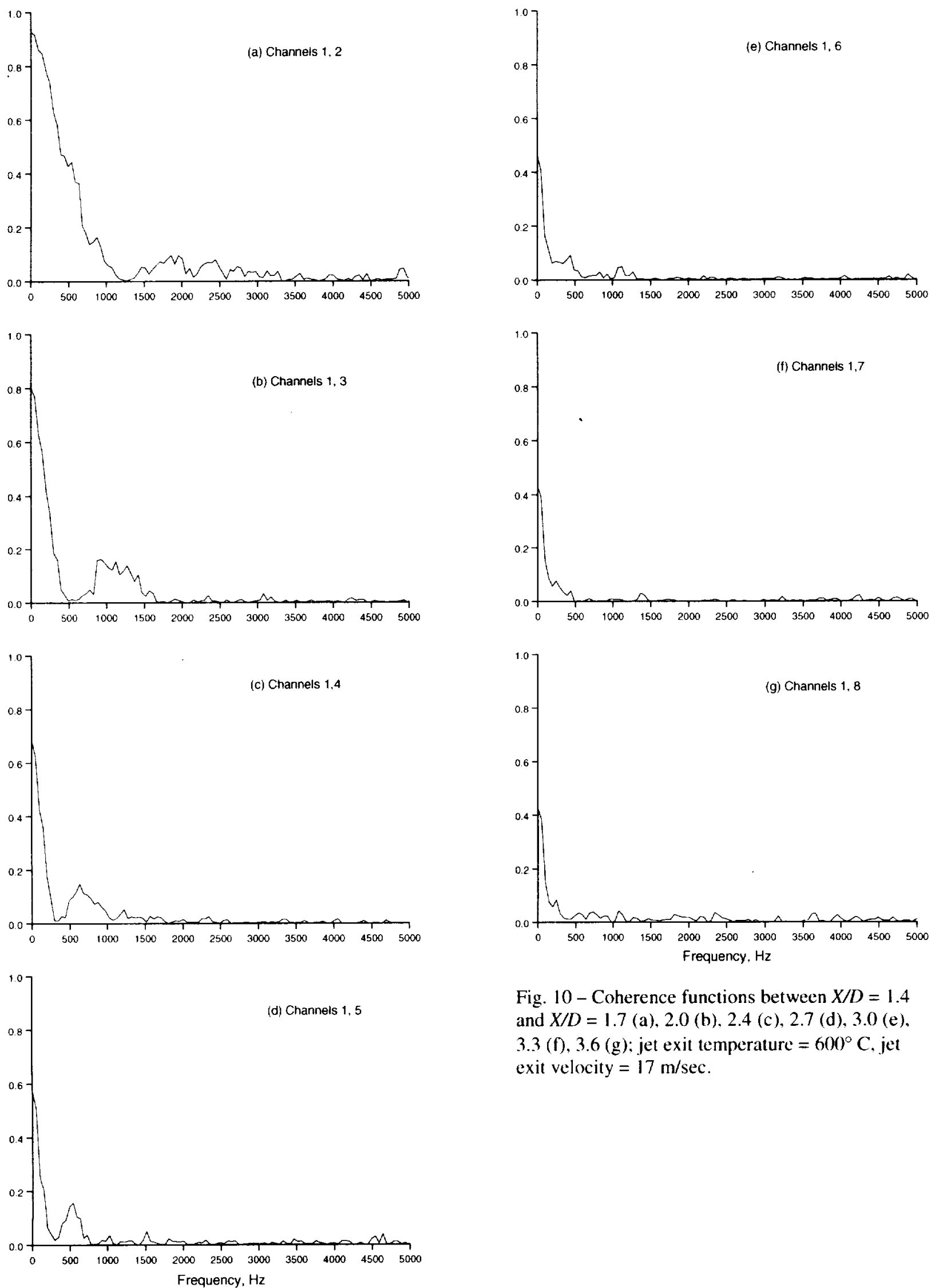

Fig. $10-$ Coherence functions between $X / D=1.4$ and $X / D=1.7$ (a), 2.0 (b), 2.4 (c), 2.7 (d), 3.0 (e), $3.3(\mathrm{f}), 3.6(\mathrm{~g})$; jet exit temperature $=600^{\circ} \mathrm{C}$, jet exit velocity $=17 \mathrm{~m} / \mathrm{sec}$. 


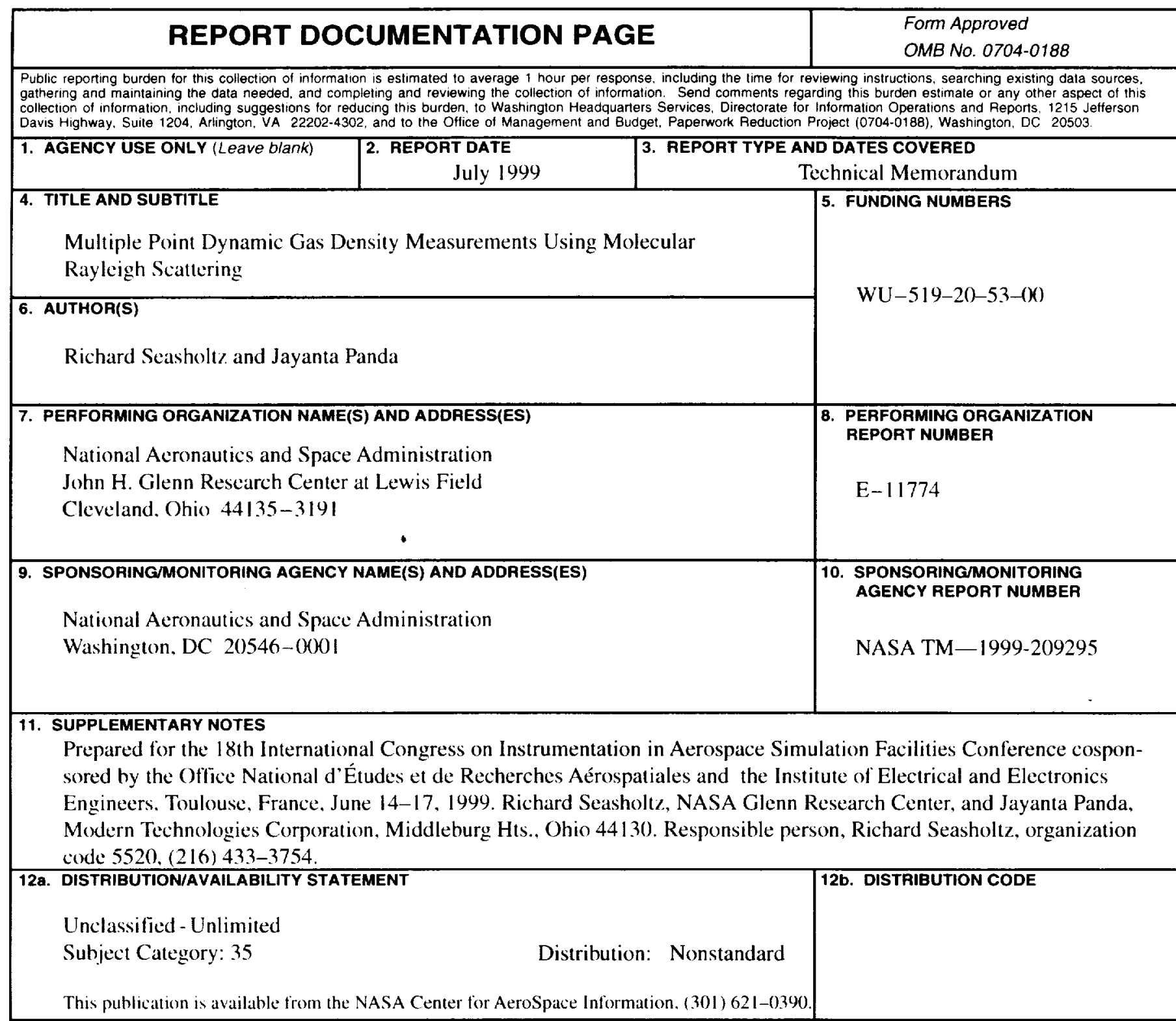

13. ABSTRACT (Maximum 200 words)

A nonintrusive technique for measuring dynamic gas density properties is described. Molecular Rayleigh scattering is used to measure the time-history of gas density simultaneously at eight spatial locations at a $50 \mathrm{kHz}$ sampling rate. The data are analyzed using the Welch method of modified periodograms to reduce measurement uncertainty. Crosscorrelations, power spectral density functions, cross-spectral density functions, and coherence functions may be obtained from the data. The technique is demonstrated using low speed co-flowing jets with a heated inner jet.

\begin{tabular}{|c|c|c|c|}
\hline \multicolumn{2}{|l|}{ 14. SUBJECT TERMS } & & $\begin{array}{c}\text { 15. NUMBER OF PAGES } \\
16\end{array}$ \\
\hline \multicolumn{3}{|l|}{ Rayleigh scattering } & 16. PRICE CODE \\
\hline $\begin{array}{l}\text { 17. SECURITY CLASSIFICATION } \\
\text { OF REPORT }\end{array}$ & $\begin{array}{l}\text { 18. SECURITY CLASSIFICATION } \\
\text { OF THIS PAGE }\end{array}$ & $\begin{array}{l}\text { 19. SECURITY CLASSIFICATION } \\
\text { OF ABSTRACT }\end{array}$ & 20. LIMITATION OF ABSTRACT \\
\hline Unclassified & Unclassified & Unclassified & \\
\hline NSN 7540-01-280-5500 & & & $\begin{array}{l}\text { andard Form } 298 \text { (Rev. 2-89) } \\
\text { scribed by ANSI Std. Z39-18 } \\
3-102\end{array}$ \\
\hline
\end{tabular}

